\title{
Abstracts presented at the Otology Section Meetings, Royal Society of Medicine, Matthew Yung Short Paper Prize, 6 March 2015, London, UK
}

Developing an intra-operative cochlear nerve monitoring technique for use during vestibular schwannoma surgery and improving hearing rehabilitation in neurofibromatosis type 2

A Kasbekar ${ }^{1}$, Y C Tam², R Carlyon ${ }^{3}$, N Donnelly ${ }^{2}$, R Macfarlane ${ }^{2}$, D Moffat ${ }^{2}$, P Axon ${ }^{2}$

From the ${ }^{1}$ Aintree University Hospital, Liverpool, ${ }^{2}$ Addenbrookes Hospital, Cambridge, and ${ }^{3}$ Medical Research Council, Cambridge

\section{Introduction}

Neurofibromatosis type 2 (NF2) results in bilateral vestibular schwannomas and eventual total deafness due to the tumours themselves or the surgery used to excise them. The use of intra-operative cochlear nerve monitoring during vestibular schwannoma surgery can help preserve the cochlear nerve anatomically and aid in the decision-making process regarding the insertion of a cochlear implant.

\section{Methods}

We describe our journey through initial experience with auditory brainstem responses (ABRs) and cochlear implant ABRs. We progressed to middle-ear electrically stimulated ABRs using a custom-made electrode during vestibular schwannoma surgery.

\section{Results}

Our technique resulted in cochlear implantation in an NF2 patient following vestibular schwannoma removal at the same sitting.

\section{Discussion}

Electrically evoked cochlear nerve monitoring has the ability to provide objective evidence of cochlear nerve function after tumour excision and aid in the decision-making process regarding hearing rehabilitation in patients who will be rendered totally deaf. This technique will improve the hearing outcomes in NF2 patients.

\begin{tabular}{lccc}
\multicolumn{4}{c}{ TABLE I } \\
\multicolumn{4}{c}{ MEAN PERFORMANCE SCORES BY GROUP } \\
\hline Measure & Expert & Trainee & Novice \\
\hline Technique score* $^{\dagger}$ & $16.10(3.63)$ & $7.19(4.73)$ & $6.83(1.97)$ \\
End product score $^{\dagger}$ & $7.40(0.96)$ & $6.06(0.82)$ & $5.42(1.11)$ \\
Injury score $^{\text {ny }}$ & $2.80(2.20)$ & $0.88(1.13)$ & $11.33(13.67)$ \\
\hline
\end{tabular}

Data represent performance scores (and standard deviations).

*Highest possible score is 24 ; ${ }^{\dagger}$ highest possible score is 13
Construct validity of the Visible Ear Simulator: a surgical teaching tool for temporal bone dissection

A Adebayo, H Khalil, M Prior

From the Derriford Hospital, Plymouth

\section{Introduction}

Current limitations with the use of cadaveric bone for training have led to the development of virtual reality simulators. However, the cost of existing simulators has been a significant barrier to widespread adoption. Subject to successful validation studies, the Visible Ear Simulator presents a significantly more cost-effective alternative.

\section{Methods}

A total of 19 participants were recruited to a prospective, observational, cross-sectional study. Participants were given 30 minutes to perform a cortical mastoidectomy. Their performance was evaluated by two independent expert raters.

Results

Performance analysis revealed that experts were rated as superior to trainees and novices, but no significant differences were observed between trainee and novice performance.

\section{Discussion}

The Visible Ear Simulator is a useful adjunct, but is presently insufficient to fully cater for ENT training needs. Its inability to reliably distinguish between trainee and novice performance suggest that further refinement, specifically with regards to three-dimensional capability, is necessary.

Dissociation of cervical and ocular vestibular-evoked myogenic potentials in patients with peripheral vestibular disorders

J S Sandhu ${ }^{1}$, J Ray ${ }^{1}$, P A Rea ${ }^{2}$

From the ${ }^{1}$ Sheffield Teaching Hospitals and ${ }^{2}$ University Hospitals Leicester

\section{Objective}

To compare cervical and ocular vestibular-evoked myogenic potentials in patients with Ménière's disease or chronic benign paroxysmal positional vertigo (BPPV).

\section{Methods}

A prospective case-control study was conducted, which contrasted the P13-N23 and N10 amplitudes of airconducted cervical and ocular vestibular-evoked myogenic potentials respectively. The study comprised 12 patients with unilateral Ménière's disease $(8$ with definite disease 
and 4 with probable disease), 12 patients with chronic BPPV and 16 normal ears.

Results

A statistically significant $(p<0.01)$ difference between ocular and cervical vestibular-evoked myogenic potential magnitudes was observed in both the Ménière's disease and BPPV populations. Furthermore, the degree of disassociation in the Ménière's disease patients' ears was related to disease severity.

\section{Conclusion}

Compared to the cervical vestibular-evoked myogenic potential, the ocular variant is more severely impacted in ears affected by Ménière's disease and BPPV. This suggests that the two tests represent different components of the otolith sensors, and supports the notion that the generators for the cervical and ocular vestibular-evoked myogenic potentials are the saccule and utricle respectively. 